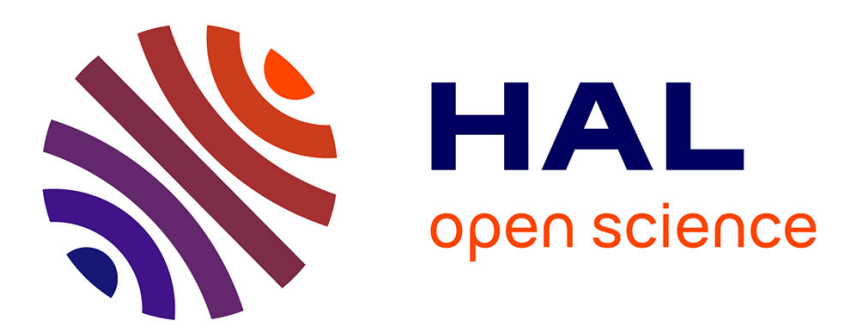

\title{
Modelling the deep drawing of a 3D woven fabric with a second gradient model
}

Gabriele Barbagallo, Angela Madeo, Fabrice Morestin, Philippe Boisse

\section{To cite this version:}

Gabriele Barbagallo, Angela Madeo, Fabrice Morestin, Philippe Boisse. Modelling the deep drawing of a 3D woven fabric with a second gradient model. Mathematics and Mechanics of Solids, 2017, 22 (11), pp.2165 - 2179. 10.1177/1081286516663999 . hal-01719711

\section{HAL Id: hal-01719711 \\ https://hal.science/hal-01719711}

Submitted on 28 Feb 2018

HAL is a multi-disciplinary open access archive for the deposit and dissemination of scientific research documents, whether they are published or not. The documents may come from teaching and research institutions in France or abroad, or from public or private research centers.
L'archive ouverte pluridisciplinaire $\mathbf{H A L}$, est destinée au dépôt et à la diffusion de documents scientifiques de niveau recherche, publiés ou non, émanant des établissements d'enseignement et de recherche français ou étrangers, des laboratoires publics ou privés. 


\title{
Modeling the deep drawing of a 3D woven fabric with a second gradient model
}

\author{
Gabriele Barbagallo ${ }^{1}$ and Angela Madeo ${ }^{2}$ and Fabrice Morestin ${ }^{3}$ and Philippe Boisse ${ }^{4}$
}

March 3, 2017

\begin{abstract}
Experimental testing on dry woven fabrics exhibits a complex set of evidences that are difficult to be completely described using classical continuum models. The aim of this paper is to show how the introduction of energy terms related to the micro-deformation mechanisms of the fabric, in particular to the bending stiffness of the yarns, helps in the modeling of the mechanical behavior of this kind of materials. To this aim, a second gradient, hyperelastic, initially orthotropic continuum theory is proposed to model fibrous composite interlocks at finite strains. In particular, the present work explores the relationship between the onset of wrinkling appearing during the simulation of the deep drawing of a woven fabric and the use of a second gradient model. It is shown that the introduction of second gradient terms accounting for the description of in-plane and out-of-plane bending rigidities, decreases the onset of wrinkles during the simulation of deep-drawing.

In this work, a quadratic energy, roughly proportional to the square of the curvature of the fibers, is presented and implemented in the simulations. This simple constitutive assumption allows to clearly show the effects of the second gradient energy on both the wrinkling description and the numerical stability of the model. The results obtained in second gradient simulations are descriptive of the experimental evidence of deep drawing whose description is targeted in this work. The present paper provides additional evidence of the fact that first gradient continuum theories alone cannot be considered fully descriptive of the behavior of dry woven composite reinforcements. On the other hand, the proposed second gradient model for fibrous composite reinforcements opens the way both to the more accurate simulation of complex forming processes and to the possibility of controlling the onset of wrinkles.
\end{abstract}

\section{Contents}

1 Introduction

2 Second gradient 3D modeling of the deep drawing 3

2.1 Second gradient modeling . . . . . . . . . . . . . . . . . . . . . . . 4

2.2 Hyperelastic initially orthotropic first gradient strain energy density . . . . . . . . . . . . . 5

2.3 Hyperelastic orthotropic second gradient strain energy density . . . . . . . . . . . . . . . 6

3 Numerical model and results $\quad 7$

3.1 Modeling geometry and contact interaction between the mold and the reinforcement . . . . 8

3.2 Augmented continuity shape functions . . . . . . . . . . . . . . . . . . . . . . 9

3.3 Influence of the second gradient on the wrinkling . . . . . . . . . . . . . . . . . . . . 10

3.4 Some considerations concerning mesh-dependency of the performed simulations . . . . . . . 12

3.4.1 First gradient model with linear shape functions . . . . . . . . . . . . . . . . . . . . 12

3.4.2 First and second gradient models with augmented continuity shape functionss . . . . . 13

3.4.3 Influence of cutting the corners on the onset of wrinkling for first and second gradient solutions . . . . . . . . . . . . . . . . . . . . . . . . 14

4 Conclusions

5 References

${ }^{1}$ Gabriele Barbagallo, gabriele.barbagallo@insa-lyon.fr, LaMCoS-CNRS \& LGCIE, INSA-Lyon, Universitité de Lyon, 20 avenue Albert Einstein, 69621, Villeurbanne cedex, France

${ }^{2}$ Angela Madeo, corresponding author, angela.madeo@insa-lyon.fr, LGCIE, INSA-Lyon, Université de Lyon, 20 avenue Albert Einstein, 69621, Villeurbanne cedex and IUF, Institut universitaire de France, 1 rue Descartes, 75231 Paris Cedex 05, France

${ }^{3}$ Fabrice Morestin, fabrice.morestin@insa-lyon.fr, LaMCoS-CNRS, INSA-Lyon, Universitité de Lyon, 20 avenue Albert Einstein, 69621, Villeurbanne cedex, France

${ }^{4}$ Philippe Boisse, philippe.boisse@insa-lyon.fr, LaMCoS-CNRS, INSA-Lyon, Universitité de Lyon, 20 avenue Albert Einstein, 69621, Villeurbanne cedex, France 


\section{Introduction}

Composite materials may possess very good characteristics due to the fact that they are obtained assembling two or more constituent materials. This kind of architectured materials can be optimized to obtain excellent resulting properties such as high strength, lightness, cost-effectiveness, thermal and electrical conductivity, insulation and many others.

In particular, the fibrous composite materials are a class of composites that is manufactured using strong fibers aligned in unidirectional sheets, non-crimped fabrics or woven fabrics (bidirectional), impregnated with a thermoset or thermoplastic resin. Woven composite materials are the most widespread choice in the case of mechanical reinforcement, due to their great formability and the subsequent possibility of designing rather complex mechanical pieces. The forming processes, such as the Resin Transfer Moulding, (RTM), have received a great deal of attention in the literature (see e.g. [46, 50, 52]). The most current forming processes consists basically of two stages:

- the dry woven fabric is preformed to obtain the desired geometry for the final part

- a thermoset resin is injected into the woven fabrics filling the pores of the fibrous reinforcement.

The process used in the forming stage is thus followed by the injection and curing of a resin in the woven fabrics, after which the finished material, union of the reinforcement and the matrix, is obtained. The quality of the obtained piece is greatly influenced by several factors, such as the characteristics of the preformed woven fabric and, in particular, its permeability, the characteristic of the resin and the temperature at which the injection process takes place. In the literature, it is possible to find a great number of articles studying in detail the injection processes and the characteristic of the resins (see $[46,50,52])$ and also the preforming processes of thin woven reinforcements (see $[11,8,9,10,13,18,19,28,31,59,60]$ ). Nonetheless, few research work concern 3D composite reinforcement forming simulation [15, 38, 47]. The focus of this paper will be on the first stage of the forming process, namely the preforming of 3D dry reinforcements. The understanding of this step is very important to determine if the preforming process is even possible. Indeed, the woven fabrics can withstand only a certain amount of shear deformation between the fibers without dissociating and an accurate modeling thus becomes crucial for optimal design.

The process of preforming can become fairly intricate when the geometries are complex (e.g. double curved geometries) and the prediction of the entirety of the properties of the deformed fabrics is, therefore, challenging. Several experimental devices have been set up to investigate the deformation modes and the possible occurrence of defects during forming of textile reinforcements [14, 35]. Among them, the hemispherical punch and die systems (Fig. 1) were especially studied because of their simple shape, double curvature and large shear angle variations between the yarns in the final state.
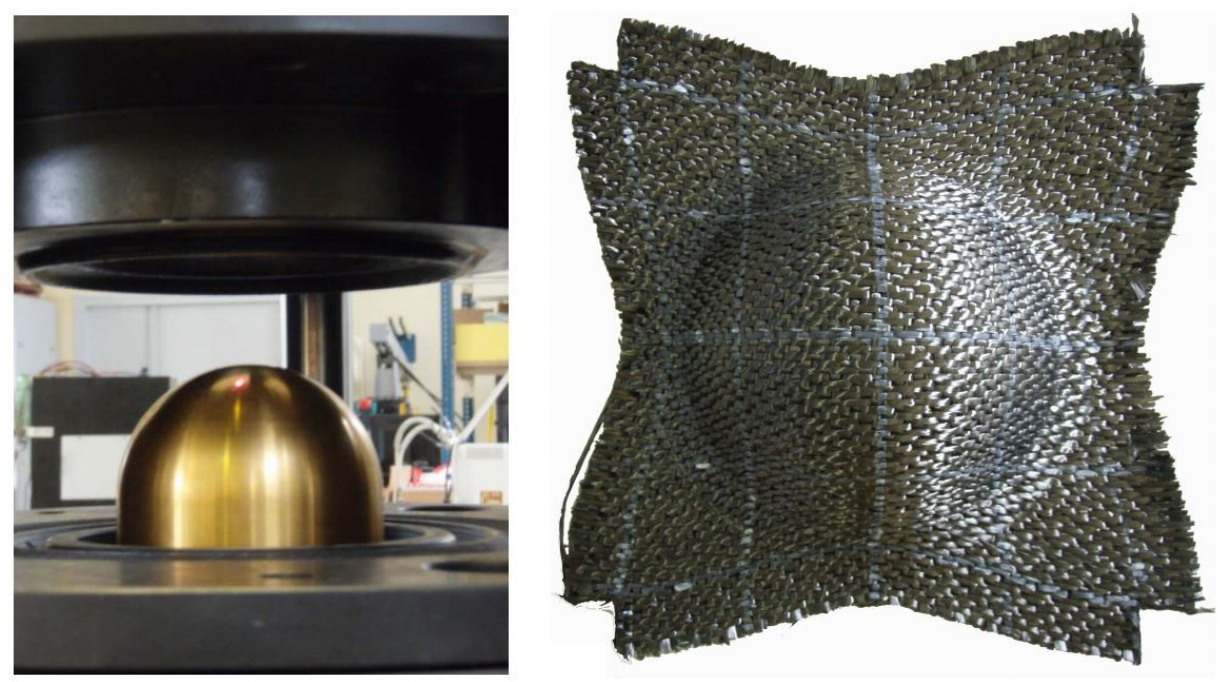

Figure 1: Experimental setup and deformation for a deep-drawing preforming with a hemispherical punch [15]. 
Reliable models for the preforming process should include information about the fiber directions and densities in the deformed state, so aiding the simulation of the resin injection and of the structural behavior of the final composite part. Indeed, the permeability of the 3D interlock fabric is strongly influenced by some deformation states that can alter or even close the interstices in the micro-structure affecting profoundly the resulting material properties. Furthermore, the direction and positioning of the yarns, that are determined solely by the preforming process, have a predominant role in the resulting mechanical properties of the composite structure (stiffness, damage and fracture, etc.).

Different approaches have been proposed to model the raw fibrous composite materials that can be found in the literature (see $[8,10,13,18,19,28,31]$ ). The most widespread approach to the simulation of fibrous composite materials is, nowadays, the finite element model that needs the determination of specific constitutive laws, to be able to describe the complex experimental evidences shown by woven composites. The interest of using second gradient theories for the more realistic description of the mechanical behavior of fibrous composite reinforcements has already been established in previous contributions (see $[6,27,36,37]$ ).

In the present paper, additional evidence is provided regarding the fact that neglecting the bending rigidities of the yarns in the modeling phase can produce inaccurate results of the simulation of 3D woven fabrics (thickness $\sim 1 \mathrm{~cm}$ ) during the modeling phase. In order to support this statement, a 3D FEM is implemented and a rather simple constitutive form of the strain energy density is introduced, accounting for:

1. initial orthotropy,

2. geometric non-linearities,

3. in-plane and out-of-plane bending of the yarns (through the introduction of suitable second gradient terms).

This second gradient model is implemented in COMSOL ${ }^{\circledR}$ looking for solutions that are continuous, as it is usual in FEM, but that also grant continuity of the first derivatives of the displacement field.

In this way, the following desirable results are obtained:

- the solution is in agreement with the observed experimental shapes (Fig. 1),

- the second gradient energy has a beneficial effect on the mesh-dependency of the solution,

- the presence of suitable second gradient terms which are descriptive of the yarns' bending allows to control the onset and evolution of wrinkles during the deep-drawing process. More particularly, if the second gradient parameter (viz the bending stiffness of the yarns) is sufficiently high, no wrinkling is observed during the simulation. This result is in agreement with the common observations of experimental results.

The results presented in this paper should be used as a guide towards the throughout implementation of FEM codes including second gradient constitutive laws for the complete modeling of the mechanical behavior of fibrous composite reinforcements during their forming process.

\section{Second gradient 3D modeling of the deep drawing}

The description of woven composites' mechanical behavior demands important efforts. Through the analysis of the deformation patterns during experimental testing, it is easy to notice that the condition of material continuity is not always strictly fulfilled, due for example to some relative slippage of warp and weft. However, if the amount of slippage between the fibers is low, a continuous model can still be used (see for example $[15,16])$. This is the approach adopted in this paper, but it must be noted that the possibility of modeling each fiber as a single detached element still exists, even if it is of difficult applicability for big mechanical pieces [23]. Continuum models with "fictive" elongations have been also introduced to account for a certain amount of slipping while remaining in a continuum framework [36].

The composite reinforcement described in the present paper is a 3D interlock fabric. It is $1 \mathrm{~cm}$ thick and it is composed of two yarn directions that are woven together and through the thickness. This 3D fabric will be analyzed with 3D Finite Elements.

The most crucial part of the continuous modeling is the definition of proper constitutive relationships that realistically reflect the mechanical properties of the analyzed material. Traditionally, the energy used in the simulation of continuous media comprises only deformations defined as first derivative of the displacement, 
so giving rise to so called first gradient theories. However, in various papers dealing with woven composites, it is shown how the addition of energies related to the local stiffness of the yarns is useful, if not necessary, to describe the macroscopic deformation behavior of the interlocks (e.g. [6, 15, 27, 37, 36]).

Considering the specific case of deep-drawing preforming, one of the phenomena which is most difficult to control with a first gradient energy is the onset of wrinkling in the deformed 3D fabric. In first gradient simulations, it is possible to observe the presence of wrinkles in the deformed configurations when a certain amount of in-plane shear stiffness is present (see for example [12]). Nevertheless, the number and amplitude of such wrinkles is a mesh-dependent phenomenon and such wrinkling is not descriptive of the experimental results. This result will be obtained again in the present paper for a traditional first gradient finite element model with linear shape functions (subsection 3.4.1).

In the remainder of the paper, it will be shown how the necessary description of the local bending effects can be performed via generalized continuum theories, such as higher order gradient or constrained micromorphic theories. More precisely, a second gradient energy, approximately proportional to the square of the yarns' curvature, is introduced and the obtained results are used to model the deep-drawing of woven fabrics.

The generalized continuum theories are still perceived as pure theoretical abstractions, even if their background was laid down in detail since the historic works of Piola [49], Cosserat [17], Midlin [42], Toupin [58], Eringen [26], Green and Rivlin [30] and Germain [29]. With the hope of a more widespread consciousness of the potential of generalized continuum theories, the authors attempt in this paper to show how some microstructure-related effects in microscopically heterogeneous mechanical systems can be still modeled by means of continuum theories.

\subsection{Second gradient modeling}

In the remainder of the paper, the following notations will be used:

- $B_{L} \subset \mathbb{R}^{3}$ is the Lagrangian or reference configuration of the considered continuum and each point $\mathbf{X} \in B_{L}$ is called a material point,

- $\chi(\mathbf{X}, t): B_{L} \times[0, T] \rightarrow \mathbb{R}^{3}$ is a suitably regular kinematic field which associates to any material point $\mathbf{X}$ its current position $\mathbf{X}$ at the time $\mathbf{t}$,

- $\mathbf{u}(\mathbf{X}, t):=\chi(\mathbf{X}, t)-\mathbf{X}$ is the displacement field at the time $\mathrm{t}$ given by the difference between the current and the reference position of each material point $\mathbf{X}$,

- $B_{E}(t)$ is the current shape of the body at any instant $t$, usually called the Eulerian configuration, given by the image of the function $\chi\left(B_{L}, t\right)$,

- the tensor $\mathbf{F}:=\nabla \chi$ is the gradient of the map $\chi$ with respect to the reference position $\mathbf{X}$,

- $\mathbf{C}:=\mathbf{F}^{T} \cdot \mathbf{F}$ is the Right Cauchy-Green deformation tensor ${ }^{5}$,

- the third order tensor field $\nabla \mathbf{C}$ is the gradient of the Cauchy-Green deformation tensor.

Compared to the first gradient models, an additional third order tensor field $\nabla \mathbf{C}$ is introduced in the xonstitutive expression of the strain energy density. This tensor field makes it possible to decribe effects related to the macro-inhomogeneity due to micro-deformations in the micro-structure of the continuum, such as the curvature of the yarns. Since second gradient theories can be readily obtained as limiting cases of micromorphic ones, it is possible to derive the second gradient contact actions in terms of the micromorphic ones following the procedure used in [7]. Some of the possible types of constraints, that could be included in such a micromorphic model which, for example, impose inextensibility of yarns so giving rise to so-called micropolar continua, are presented in $[3,24,25,26,48]$.

A hyperelastic, initially orthotropic, second gradient model can be applied to the case of thin fibrous composite reinforcements at finite strains. For the strain energy density $W(\mathbf{C}, \nabla \mathbf{C})$, which will be used to simulate the mechanical behavior of the fibrous materials in the finite strain regime, it is assumed a decomposition such as:

$$
W(\mathbf{C}, \nabla \mathbf{C})=W_{I}(\mathbf{C})+W_{I I}(\nabla \mathbf{C}),
$$

\footnotetext{
${ }^{5}$ A central dot indicates simple contraction between tensors of order greater than zero. For example if $\mathbf{A}$ and $\mathbf{B}$ are second order tensors of components $A_{i j}$ and $B_{j h}$ respectively, then $(\mathbf{A} \cdot \mathbf{B})_{i h}:=A_{i j} B_{j h}$, where Einstein notation of sum over repeated indexes is used.
} 
where $W_{I}$ is the first gradient strain energy and $W_{I I}$ is the second gradient one. The specific constitutive forms of the first and second gradient strain energy densities used to model fibrous composite reinforcements are explicitly presented in the following subsections.

\subsection{Hyperelastic initially orthotropic first gradient strain energy density}

Even at finite strains, well-known expressions for isotropic strain energies descriptive of the behavior of isotropic materials are available in the literature (see e.g. $[44,56]$ ). Quite the opposite happens in the case of orthotropic materials, for which suitable specific strain energies, well descriptive of real material behaviors are more difficult to be found. Some results are provided in [34], where some polyconvex energies are proposed to describe the deformation of rubbers in uniaxial tests. Explicit anisotropic hyperelastic potentials for soft biological tissues are also proposed in [33] and reconsidered in [5, 53], in which their polyconvex approximations are derived. Other examples of polyconvex energies for anisotropic solids are given in [57].

Notwithstanding the research efforts devoted to the study of polyconvexity, which certainly introduce rigorous theoretical frameworks for the study of the mechanical behaviors of hyperelastic materials, the use of such polyconvex models is often limited due to the difficult attribution of a sensible physical meaning to the wealth of constitutive parameters which are introduced. The approach adopted in this paper is the Ockham's razor approach, introducing the minimum possible number of physically sensible constitutive parameters which are needed to describe the targeted phenomena.

In the literature, reliable constitutive models for the description of the mechanical behavior of fibrous composite reinforcements at finite strains can be found in $[1,15,16]$. Moreover, the mechanical behavior of composite preforms with rigid organic matrix (see e.g. [40,41, 45]) is quite different from the behavior of the sole fibrous reinforcements (see e.g. [15]) rendering the mechanical characterization of such materials a major scientific and technological issue.

We start by specifying the expression of the work of internal actions which is suitable to describe the deformation of the considered system. To do so, we start by recalling classical results for first gradient, hyperelastic orthotropic continua which prescribe the functional dependence that the strain energy density of an orthotropic continuum must have on the Cauchy-Green strain tensor $C=F^{T} \cdot F$ (see e.g. [?, ?, 16, ?, $34, ?, 44, ?, 51, ?, 56])$.

Here and in the sequel, we denote by $\chi: B \rightarrow \mathbb{R}^{3}$ the placement function associated to the considered body that associates to any material particle $X \in B$ its current position $x$ in the deformed configuration and which can be related to the displacement field by means of the relation $\chi=u+X$. Moreover, we denote by $F=\nabla \chi$ the space gradient of the introduced placement field.

We start by assuming that the strain energy density can be given in the form

$$
W(C, \nabla \varphi)=W_{I}(C)+W_{I I}(\nabla \varphi) .
$$

Representation theorems for 3D orthotropic first gradient materials are available in the literature (see e.g. $[51, ?, ?])$, which state that the functional dependence of the strain energy density on the strain tensor $C$ must be given in terms of its invariants $i_{O}:=\left\{i_{1}, i_{4}, i_{6}, i_{8}, i_{9}, i_{10}\right\}$, where the introduced invariants are defined in table 1 in which also their physical interpretation can be found.

Explicit expressions for the strain energy potential as function of the invariants $i_{O}$ which are suitable to describe the real behavior of orthotropic hyperelastic materials are difficult to be found in the literature. Certain constitutive models are for instance presented in [34], where some polyconvex energies for orthotropic materials are proposed to describe the deformation of rubbers in uniaxial tests. Explicit anisotropic hyperelastic potentials for soft biological tissues are also proposed in [33] and reconsidered in $[5,53]$ in which their polyconvex approximations are derived. Other examples of polyconvex energies for anisotropic solids are given in [57]. It is even more difficult to find in the literature reliable constitutive models for the description of the real behavior of fibrous composite reinforcements at finite strains but some attempts can be for instance recovered in $[1,16]$. Furthermore, the mechanical behavior of composite preforms with rigid organic matrix (see e.g. [22, 41, 40, 45]) is quite different from the behavior of the sole fibrous reinforcements (see e.g. [15]) rendering the mechanical characterization of such materials a major scientific and technological issue. 


\begin{tabular}{|c|c|c|}
\hline Invariant & Expression & Meaning in terms of deformation \\
\hline \hline$i_{1}$ & $\operatorname{tr}(C)$ & Averaged changes of length \\
\hline$i_{4}$ & $m_{1} \cdot C \cdot m_{1}$ & Local stretch in the direction $m_{1}$ \\
\hline$i_{6}$ & $m_{2} \cdot C \cdot m_{2}$ & Local stretch in the direction $m_{2}$ \\
\hline$i_{8}$ & $m_{1} \cdot C \cdot m_{2}$ & Angle variation between the directions $\left(m_{1}, m_{2}\right)$ \\
\hline$i_{9}$ & $m_{1} \cdot C \cdot m_{3}$ & Angle variation between the directions $\left(m_{1}, m_{3}\right)$ \\
\hline$i_{10}$ & $m_{2} \cdot C \cdot m_{3}$ & Angle variation between the directions $\left(m_{2}, m_{3}\right)$ \\
\hline
\end{tabular}

Table 1: Invariants of the Green-Lagrange strain tensor in the orthotropic case. The vectors $m_{1}$ and $m_{2}$ are unit vectors in the two privileged directions of the material and $m_{3}:=m_{1} \times m_{2}$.

In this work, the directions $\mathbf{D}_{1}$ and $\mathbf{D}_{2}$ denote the unit vectors in the directions of the warp and weft yarns in the reference configuration and the direction $\mathbf{D}_{3}=\mathbf{D}_{1} \times \mathbf{D}_{2}$ denotes the unit normal to the plane containing the two sets of fibers. It is possible to fully describe a first gradient orthotropic energy with an expression of the type (see e.g. [51]):

$$
W_{I}(\mathbf{C})=W_{I}\left(i_{11}, i_{22}, i_{33}, i_{12}, i_{13}, i_{23}\right),
$$

where $i_{i i}=\mathbf{D}_{i} \cdot \mathbf{C} \cdot \mathbf{D}_{i}, i=\{1,2,3\}$ represents the elongation strain in the direction $\mathbf{D}_{i}$ and $i_{i j}=\mathbf{D}_{i} \cdot \mathbf{C} \cdot \mathbf{D}_{j}$ represents the shear strain (angle variation) between the directions $\mathbf{D}_{i}$ and $\mathbf{D}_{j}$ with $i, j \in\{1,2,3\}$ and $i \neq j$.

It is possible to develop complex non-linear energies that capture in all details the mechanical nonlinearities observable in the experimental testing, as done in $[1,15,16]$, but, as already pointed out, this is not one of the aims of the present paper. Instead, using only a simple quadratic first gradient energy, it is possible to thoroughly analyze the influence of both meshing and additional second gradient terms on the performed numerical simulations. Thus, the chosen constitutive expression for the first gradient energy is:

$$
W_{I}(\mathbf{C})=\frac{1}{2} K_{11}\left(\sqrt{i_{11}}-1\right)^{2}+\frac{1}{2} K_{22}\left(\sqrt{i_{22}}-1\right)^{2}+\frac{1}{2} K_{33}\left(\sqrt{i_{33}}-1\right)^{2}+\frac{1}{2} K_{12} i_{12}^{2}+\frac{1}{2} K_{13} i_{13}^{2}+\frac{1}{2} K_{23} i_{23}^{2},
$$

where $K_{i i}$ are the extensional stiffnesses in the direction of the yarns as well as in the orthogonal direction, while $K_{i j}$ with $i \neq j$ are the in-plane and out-of plane shear stiffnesses. The numerical values of the material parameters were chosen to define a material in which the extensional stiffness is much higher than the shear stiffness and the shear behavior in the plane of the fibers is stiffer than the out of plane. Moreover, the extensional stiffness in the orthogonal plane is much lower than the in-plane ones, due to the fact that no yarns are effectively present in the thickness of the interlock. Even if more refined hyperelastic laws can be certainly be introduced in the spirit of $[1,15,16]$, the proposed expression for the first gradient energy density is representative of the main macroscopic deformation modes of fibrous interlocks. The parameters chosen are the ones shown in Tab. 2.

\begin{tabular}{|c|c|c|c|c|c|}
\hline$K_{11}$ & $K_{22}$ & $K_{33}$ & $K_{12}$ & $K_{13}$ & $K_{23}$ \\
\hline \hline $5 \mathrm{MPa}$ & $5 \mathrm{MPa}$ & $0.5 \mathrm{MPa}$ & $50 \mathrm{kPa}$ & $0.5 \mathrm{kPa}$ & $0.5 \mathrm{kPa}$ \\
\hline
\end{tabular}

Table 2: Parameters of the first gradient energy.

\subsection{Hyperelastic orthotropic second gradient strain energy density}

Considering linear elastic isotropic second gradient media, it is possible to find constitutive laws that are able to describe a very wide set of behaviors (see for example [20]). In the case of the woven fabrics, the bending stiffness of the yarns is the main micro-structure-related deformation mechanism which takes place at the mesoscopic level and, therefore, it is the only one that will be considered in this paper. The modeling of the bending stiffness of the yarns is decisive for the description of some specific phenomena, such as shear transition layers in 2D experimental tests and wrinkling during the deep-drawing of dry woven fabrics. A second gradient theory is potentially able to account for other effects related to the derivatives of the elongations but, in this work, they will be disregarded. The second gradient energy considered is, thus, function only of the derivatives of the invariants $i_{i j}(i \neq j)$, that can be used to define rough descriptors of the curvatures of the two sets of yarns of the fabric. 
As a matter of fact, it can be inferred (see also $[6,21,27,36,37]$ ) that, given the family of yarns initially oriented in the direction $\mathbf{D}_{1}$, the quantity $i_{12,1}$ is a measure of their in-plane bending ${ }^{6}$. Analogously $i_{12,2}$ is a measure of the in-plane bending of the family of yarns initially oriented in the direction $\mathbf{D}_{2}$. The quantities $i_{13,1}$ and $i_{23,2}$ are descriptors of the out-of-plane bending of the yarns initially oriented in the $\mathbf{D}_{1}$ and $\mathbf{D}_{2}$ directions, respectively. Since no material fibers are present in the thickness of the considered interlocks, quantities related to their bending $\left(i_{13,3}\right.$ and $\left.i_{23,3}\right)$ are not likely to play a role in the deformation of such materials. In the light of these remarks, the following constitutive form is introduced for the second gradient strain energy density:

$$
W_{I I}(\nabla \mathbf{C})=\frac{1}{2} \alpha_{1} i_{12,1}^{2}+\frac{1}{2} \alpha_{2} i_{12,2}^{2}+\frac{1}{2} \beta_{1} i_{13,1}^{2}+\frac{1}{2} \beta_{2} i_{23,2}^{2},
$$

where with $\alpha_{1}, \alpha_{2}$ and $\beta_{1}, \beta_{2}$ are the in-plane and out-of-plane bending stiffnesses of the two family of yarns, respectively. For unbalanced fabrics, i.e. fabrics whose warp and weft yarns do not have the same characteristics, it is likely that $\alpha_{1} \neq \alpha_{2}$ and $\beta_{1} \neq \beta_{2}$ (see also $[6,36]$ ). The object of this paper are interlocks which are balanced and, hence, it is assumed that $\alpha_{1}=\alpha_{2}=\alpha$ and $\beta_{1}=\beta_{2}=\beta$. Moreover, it is possible that the two families of yarns have different bending stiffnesses in-plane and out of plane. Nevertheless, such difference can be rather small, so in this paper it will also be set $\alpha=\beta$. The chosen second gradient energy thus takes the particular form:

$$
W_{I I}(\nabla \mathbf{C})=\frac{1}{2} \alpha\left(i_{12,1}^{2}+i_{12,2}^{2}+i_{13,1}^{2}+i_{23,2}^{2}\right),
$$

Further investigations are needed to establish a strict theoretical relationship between the microscopic structure of considered reinforcements and the macroscopic parameters here introduced: it is indeed well known that the second gradient parameters are intrinsically related to a characteristic length $L_{c}$ which is, in turn, associated to the micro-structural properties of considered materials. Many identification methods have been introduced to relate the macroscopic second gradient parameter to the microscopic properties of the considered medium, e.g. see [2, 54]. Suitable multi-scale methods as the one introduced in [43] may be generalized to be applied to the present case. Moreover, the description of the considered system at the microscopic scale may exploit some of the results proposed in $[4,32,55]$.

\section{$3 \quad$ Numerical model and results}

In this section, the implemented FEM and the consequent results for the simulation of deep-drawing are shown.

The structure of this section will be as follows:

- in the first subsection, the numerical implementation of the contact interaction between the testing machine and the interlock is presented,

- in the second subsection the proposed constrained shape functions, that will be here called augmented continuity shape functions and used to perform a numerical simulation of the presented second gradient model, will be defined,

- in the third subsection, the influence of the second gradient parameter $\alpha$ on the onset of wrinkles during the simulation of the deep-drawing is studied,

- the fourth subsection presents some observed the mesh-dependency result for the first gradient model, when using linear shape functions,

- the final subsection shows the first and second gradient solutions as functions of the adopted mesh, when using the augmented continuity shape functions. It is concluded that second gradient simulations are not significantly affected by the choice of the mesh, provided that the size of the elements is sufficiently small.

\footnotetext{
${ }^{6}$ Here and in the sequel the term $(\cdot)_{, i}$ denotes the partial derivative of the quantity $(\cdot)$ with respect to the space coordinates $\xi_{i}$ of a reference frame oriented within the directions $\mathbf{D}_{i}$.
} 


\subsection{Modeling geometry and contact interaction between the mold and the re- inforcement}

The object of the paper is the simulation of the deep-drawing process performed on 2.5D composite interlocks. In particular, the focus will be on a hemispherical punch and dye system, as the one shown in Fig. 2. In such test, a square dry woven composite interlock is formed by an hemispheric punch that, with the presence of a horizontal plane makes the deformed shape assume a double-curvature shape.

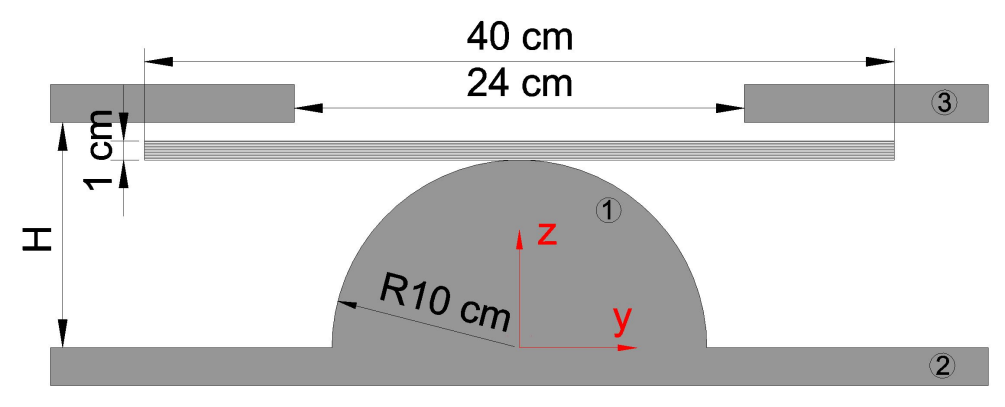

Figure 2: Geometry of the model for a deep-drawing preforming with a hemispherical punch.

To implement the contact between the woven composite and the testing machine, a penalty function was introduced such that, to each interpenetration, it associates a stress $\mathbf{t}$ normal to the surface to be applied on the fabric, in formulas:

$$
\mathbf{t}=K_{\text {contact }} \Delta \mathbf{n},
$$

where $K_{\text {contact }}$ is an opportune stiffness that is set to increase in each non-linear iteration so as to obtain the minimum interpenetration possible, $\Delta$ is the interpenetration between the woven fabrics and the machine, and $\mathbf{n}$ is the normal to the surface of the punch or of the die. The perpendicularity of the assumed stress is equivalent to assume that no friction is present between the experimental setup and the specimen. This could be considered a strong hypothesis, but the results seem to be qualitatively correct and this suffices for the purposes of this paper.

In the presented model, it was chosen to model the punch and the die as rigid bodies since they are supposed to have a stiffness of various orders of magnitude higher than the specimen. Thus, the position of the die and of the punch is known a priori in each of the steps of the test, leading to a much easier determination of the contact stresses in Eq. (7). Indeed, considering as origin of the reference system the center of the basis of the hemispherical punch, the $\mathbf{Z}$ axis as the vertical loading direction and the current position $(x, y, z)$ of a point of the fabric, the interpenetrations between the woven fabric and the hemisphere, the lower plane and the die, respectively, can be expressed as:

$$
\Delta_{1}=\max \left(R-\sqrt{x^{2}+y^{2}+z^{2}}, 0\right), \quad \Delta_{2}=\max (-z, 0), \quad \Delta_{3}=\max \left(z-H+w_{0}, 0\right),
$$

where $R$ is the radius of the hemispherical punch, $H_{0}$ and $w_{0}$ are the initial position and the applied displacement of the die in the considered step, respectively. The direction of the resulting stress is radial for the hemispherical punch and vertical for the plane and the die. Therefore, considering once again the center of the hemispherical punch as the origin of the reference system, it is possible to write:

$$
\mathbf{n}_{1}=\frac{(x, y, z)}{\sqrt{x^{2}+y^{2}+z^{2}}}, \quad \quad \mathbf{n}_{2}=(0,0,1), \quad \mathbf{n}_{3}=(0,0,-1),
$$

The resulting stresses $\mathbf{t}_{1}$ and $\mathbf{t}_{2}$ were applied to the lower surface of the specimen, while $\mathbf{t}_{3}$ was applied to the upper surface. Finally, the resulting contact stresses can be written as:

$$
\begin{aligned}
& \mathbf{t}_{1}=K_{\text {contact }} \max \left(\frac{R}{\sqrt{z^{2}+y^{2}+z^{2}}}-1,0\right)(x, y, z), \\
& \mathbf{t}_{2}=K_{\text {contact }} \max (-z, 0)(0,0,1), \\
& \mathbf{t}_{3}=K_{\text {contact }} \max \left(z-H+w_{0}, 0\right)(0,0,-1) .
\end{aligned}
$$




\subsection{Augmented continuity shape functions}

As it is well known, the usual steps followed in finite element models are as follows:

1. Discretize the considered body $\Omega$ with a mesh. Each of the obtained sub-bodies $\Omega_{e}$ is called an element.

2. In each element $\Omega_{e}$, introduce a suitable number $N_{n}$ of local nodes $\left\{x_{1}^{e}, x_{2}^{e}, \ldots, x_{N_{n}}^{e}\right\}$ which are needed to define a basis of polynomials $\left\{f_{1}^{e}, f_{2}^{e}, . ., f_{N_{n}}^{e}\right\}$, of order n, which will be subsequently used to build-up the solution. Of course, the number of points $N_{n}$ to be introduced depends on the order of the chosen polynomials (e.g. for $1 \mathrm{D}$ elements $N_{n}=n+1$ ). The polynomials $f_{i}^{e}$ are given and are built in such a way that:

$$
\left\{\begin{array}{l}
f_{i}^{e}\left(x_{j}^{e}\right)=0, \quad j \neq i, \\
f_{i}^{e}\left(x_{i}^{e}\right)=1, \quad i, j \in 1,2, \ldots, N_{n} \\
f_{i}^{e}=0, \quad \text { outside the element } \Omega_{e}
\end{array}\right.
$$

3. Look for a global solution that takes the form:

$$
u=\sum_{e=1}^{N_{e}} \sum_{i=1}^{N_{n}} a_{i}^{e} f_{i}^{e},
$$

where $N_{e}$ is the total number of elements $\Omega_{e}$ of the chosen discretization of $\Omega$, and $a_{i}^{e}$ are constants to be determined. Clearly, the number of constants $a_{i}^{e}$ depends both on the number of elements and on the order $n$ of the chosen polynomials $f_{i}^{e}$.

The more common finite elements codes, such as the one used to perform the simulations in this paper, are built in such a way that the unknown constants are determined according to the following procedure:

1. calculate some $a_{i}^{e}$ by means of the imposed boundary conditions (e.g. assigned displacement at a boundary node),

2. calculate some others $a_{i}^{e}$ by imposing continuity of the field $u$ through the elements $\Omega_{e}$,

3. calculate the remaining constants $a_{i}^{e}$ by means of a suitable minimization of the global action functional $\mathcal{A}$ associated to the energy $\mathrm{W}$.

Such elements are usually built to treat problems in classical elasticity where first gradient energies $W(\nabla u)$ are introduced. Note that the global continuity of displacement is imposed a priori in these elements.

The physical phenomena, object of this paper, typically show deformed shapes that exhibit continuity of displacement and also of strain (first derivatives of displacement). Indeed, from the experimental observations, it is possible to notice that, even if there are rapid changes of the strain within the specimen, they are always smoothened by the presence of transition layers allowing to pass from a value of strain to the other in a continuous way (see e.g. $[6,27,36]$ ).

To describe the considered phenomena, namely continuous displacements and rapid but continuous variation of strain, two ingredients are needed:

1. the introduction of a second gradient energy, e.g. of the type in Eq. (6),

2. an adapted finite element which is able to assure the continuity of the first derivatives of displacement (class of continuity $C^{1}$ ).

Such second point can be achieved at least in two ways:

1. introducing a supplementary kinematical field $P: \Omega \rightarrow \mathbb{R}^{3}$, subsequently constrained to be related to first derivatives of displacement as $P \rightarrow\left(i_{12}, i_{13}, i_{23}\right)$ e.g. using Lagrange multipliers or penalty methods (e.g. see [36, 37]),

2. keeping the same kinematics (only the standard displacement field) and try to force the finite element to grant continuity of first derivatives of displacement. 
In this paper, the authors chose to implement this second way of granting continuity of strain by introducing third order Lagrangian polynomials which guarantee such augmented continuity with a penalty energy at the element interfaces of the type ${ }^{7}$ :

$$
W_{\text {Interface }}=K_{\text {Penalty }}\left(\left[\left[i_{12}\right]\right]^{2}+\left[\left[i_{13}\right]\right]^{2}+\left[\left[i_{23}\right]\right]^{2}\right) .
$$

This energy depends only on the discontinuity of the deformations $i_{12}, i_{13}$ and $i_{23}$ and it is, therefore, not sufficient to render the entire $\nabla u$ continuous. Nonetheless, the derivatives of the deformations $i_{12}, i_{13}$ and $i_{23}$ are the only ones appearing in the presented second gradient energy and, therefore, are the only ones on which the continuity is imposed.

Whit this workaround it was possible to obtain almost continuous deformations and, thus, to implement directly a second gradient 3D model. The possibility of adding an energy on the interface between mesh elements is not always granted, but it is possible in COMSOL ${ }^{\circledR}$, the software used for the simulations presented in the present paper.

\subsection{Influence of the second gradient on the wrinkling}

The model here presented implements the augmented continuity shape functions in a COMSOL ${ }^{\circledR}$ finite element model. The energy considered was the sum of the first gradient energy presented in Eq. (4) and of the second gradient one given in Eq. (6), for which the directions of the fibers $\mathbf{D}_{1}$ and $\mathbf{D}_{2}$ were chosen to be parallel to the edges of the specimen. The first gradient parameters are the ones shown in the Tab. 2, while various values of the second gradient parameter $\alpha$ were considered. It must be noted that, in the case $\alpha=0$, the model reduces to a first gradient model with the energy in Eq. (4).

The results, obtained for $\alpha=0,0.1,1,10 N$, are shown in Fig. 3 for an imposed displacement of $90 \%$ of the punch's radius $(9 \mathrm{~cm})$. In the first gradient case, it is possible to notice the presence of a significant number of wrinkles in the fibers direction causing a considerable out-of-plane curvature of the fibers. Instead, the insertion of a second gradient energy depending on the curvature generates a tendency to reduce the wrinkling effect. If the value of $\alpha$ increases to the value $10 N$ all the secondary wrinkling disappears and the only principal wrinkle remaining is due to the natural evolution of the double curvature of the macroscopic configuration.

\footnotetext{
${ }^{7}$ The term [[-]] denotes the jump of the quantity - at the interface between two elements $\Omega_{e}$.
} 


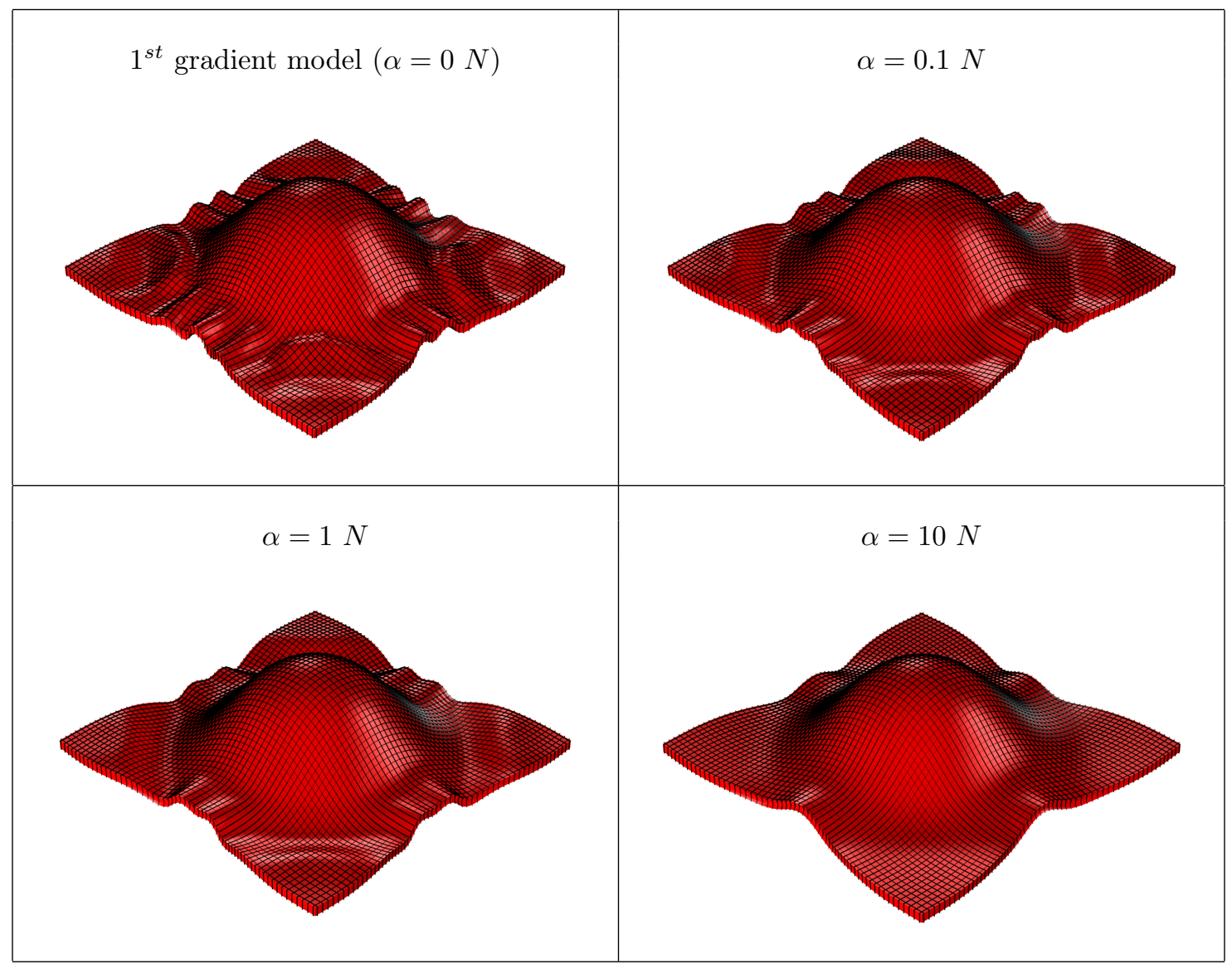

Figure 3: Dependence of the solution on the second gradient parameter $\alpha$.

As it will be shown in subsections 3.4.1 and 3.4.2, the first gradient model appears to be mesh-dependent even after the introduction of the augmented continuity shape functions (see Fig. 6) and it is, therefore, impossible to show a representative solution for this case (see subsections 3.4.1 and 3.4.2). Thus, it was chosen to show the deformed shape evaluated with the thinner used mesh, even if it is reasonable to assume that more wrinkles could appear for thinner meshes. On the other hand, even for small values of the second gradient parameter $\alpha$, a stabilization of the deformed configuration is obtained (see Fig. 6) and the deformed shape presented can be considered a representative solution, as it will be shown in subsections 3.4.2.

The possibility of controlling the onset and evolution of wrinkling during the deep-drawing simulation via the introduction of a constitutive parameter could be of great use in the prevision of the material behavior in view of structure design. It must be reminded that the presented simulations are relative to an experimental test that is meant for the characterization of the material constitutive properties. It is, therefore not enough to correctly describe the experimental results but the final goal is to predict the behavior of the woven fabric in generic engineering applications.

An issue that has to be covered is the determination of the second gradient parameter via experimental testing. Considering the proposed simple energy, it could be possible to heuristically choose $\alpha$ in order to have a qualitative description of the wrinkling phenomenon during a test such as the one proposed here. Furthermore, there are several experimental phenomena whose description would be useful for the calibration of a second gradient energy. During a Bias Extension Test, it is possible to observe the formation of some shear boundary layers the description of which can be used to calibrate the second energy parameters, as shown in [27]. In the case of a Bias Extension Test on strongly unbalanced fabrics, the bending stiffness of the fibers can lead to some macroscopic effects like the asymmetric deformed shape analyzed in $[6,36]$. Finally, the calibration of the second gradient parameter could be attempted via a three point bending of an interlock, as in [37]. Which combination of these tests is best suited for the determination of the second gradient parameters is still to be decided, but it is important to have multiple observable effects so that it is possible to validate the chosen parameters.

The results obtained in this paper are a confirmation of the great potential of the use of a second gradient 
model for the description of the wrinkling phenomenon and, more generally, of the behavior of composite materials. In the authors' opinion, the results presented in this paper and in $[6,27,36,37]$ are starting to clearly show how a second gradient model can be a potential solution for most of the issues relative to the description of the behavior of dry woven fibrous composite.

\subsection{Some considerations concerning mesh-dependency of the performed simu- lations}

\subsubsection{First gradient model with linear shape functions}

As stated above, the results obtained via a first gradient model appear to be mesh-dependent, due to the non-stability of the wrinkling description. The aim of this subsection is to present this issue in the case of a classical first gradient implementation and, hence, to show the stabilization effect obtained with the insertion of a second gradient energy.

The augmented continuity shape functions introduced for the second gradient model are momentarily discarded, so that it is possible to frame the mesh-dependency problem in a more traditional setting. In the simulations of this subsection, it was chosen to implement a model with the Lagrange linear shape functions. The study of the mesh-density's influence on the first gradient solution is made with two types of mesh, namely:

- hexahedral meshes obtained sweeping quadrilateral meshes on the boundary over the thickness of the specimen (Fig. 4),

- tetrahedral elements (Fig. 5).

The same results cannot be obtained for the second gradient model because with such low continuity shape functions the insertion of a second gradient energy cannot be detected and, hence, it plays no actual role in the results.

In Fig. 4, the hexahedral meshes and the resulting deformed shapes of the specimen are shown. It is important to remark that in this set of meshes the directions of the yarns $\mathbf{D}_{1}$ and $\mathbf{D}_{2}$, that are parallel to the edges of the specimen, coincide with the normals to the mesh interfaces. This property makes it possible to have a discontinuity on the derivatives in one of the fiber directions without losing the smoothness in the other direction. In other words, it is possible to form a wrinkle at the element interfaces for one set of fibers keeping the other set of fiber unaffected. This uncoupling can cause the formation of several wrinkles without interfering in other deformation mechanisms. Being the effect strongly related to the positioning and number of interfaces between meshes, it is not surprising that the result appears to be mesh-dependent. As a matter of fact, it is possible to see in Fig. 4 how the increase in mesh-density seems to be connected to an increment in the number of wrinkles.

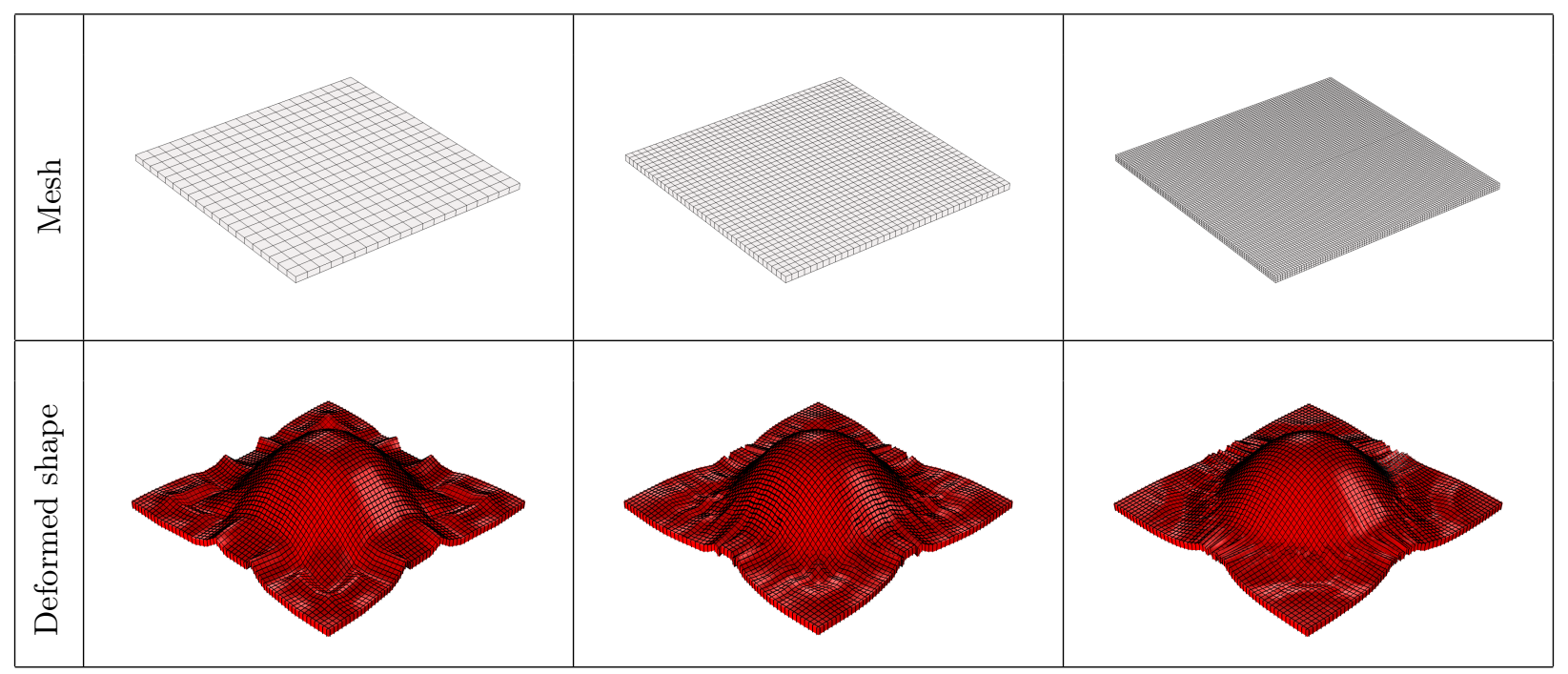

Figure 4: Solution of a first gradient model with linear shape functions and hexahedral meshes of different sizes. 
Changing the type of mesh to tetrahedral elements as shown in Fig. 5, the improvement obtained in the stability is very clear. Despite the solution is once again mesh-dependent, the differences obtained in the output are much less with respect to the hexahedral mesh. The explanation for this result is that, in this case, the normals to the interfaces between the meshes do not always coincide with the direction of the fibers making the appearance of a wrinkling phenomenon at the interfaces more difficult.

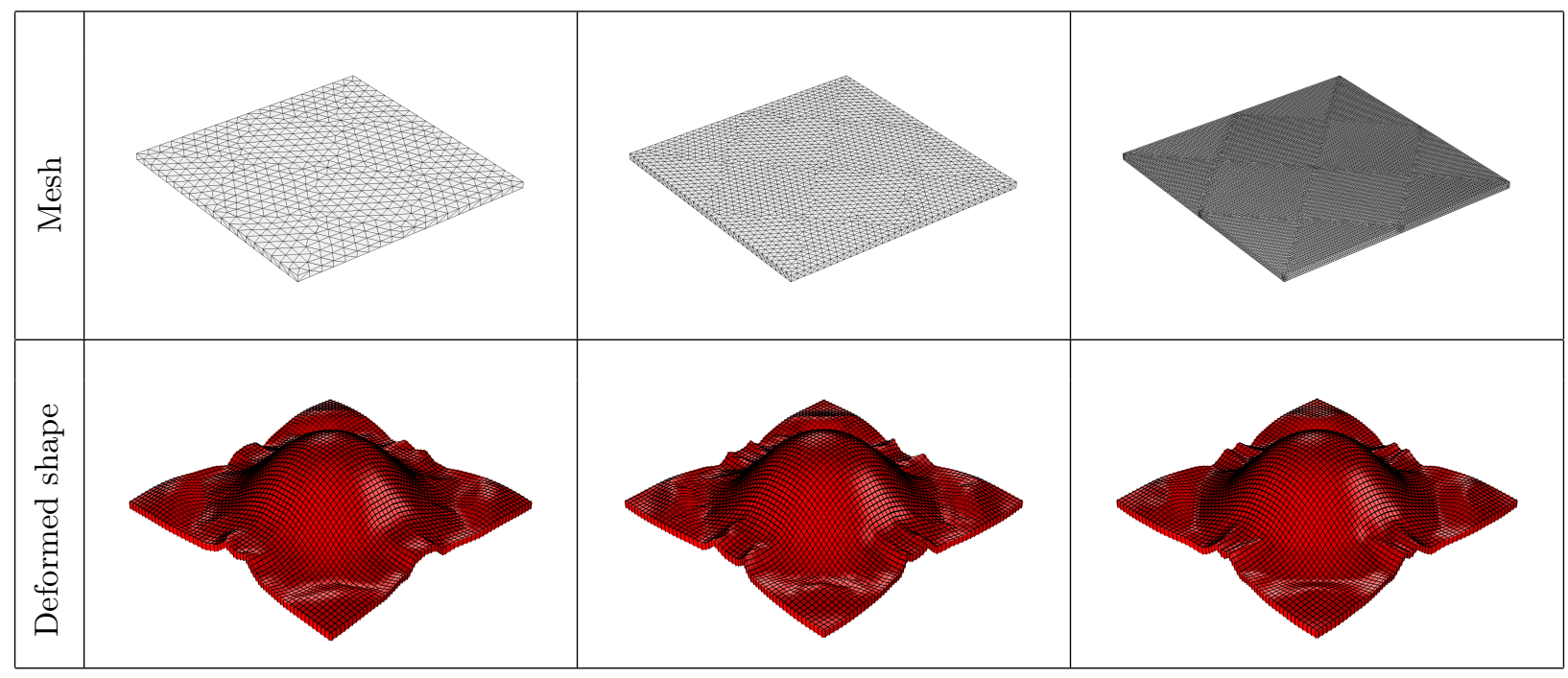

Figure 5: Solutions for a first gradient model with linear shape functions and tetrahedral elements of different sizes.

The conclusion on the results presented in this subsection is that a first gradient model with linear shape function may present unphysical wrinkling phenomena. It is still possible to obtain realistic results from such a model, but the dimension and the orientation of the elements should be carefully chosen to avoid unphysical wrinkling.

\subsubsection{First and second gradient models with augmented continuity shape functionss}

In this subsection, a study of first and second gradient solutions obtained considering augmented continuity shape functions is presented in Fig. 6. At first glance, it could seem that the mesh considered is poorer with respect to the previous case, but with the third degree polynomials used as shape function the number of nodes is comparable to the linear case.

It is possible to see that the first gradient solution still seems to depend on the size of the mesh. The wrinkles are not spikes corresponding to an interface between two mesh-elements, as happened in Fig. 4, because the augmented continuity shape functions impose the smoothness of the strain during the deformation process.

Fig. 6 explicitly shows that the stability of the model seems to be increased by adding a second gradient energy. Indeed, the wrinkling phenomenon is controlled by the second gradient terms and the corresponding result appears to be mesh-independent even with a small constitutive parameter $(\alpha=0.1 N)$. The fact that second gradient terms stabilize the numerical onset of wrinkling so producing more realistic results is not surprising. Indeed, the presence of an out-of-plane bending stiffness of the yarns (which is of course evident from a phenomenological point of view) makes energetically expensive the formation of wrinkles. On the other hand, since no energetic cost is associated to out-of-plane bending withing first gradient theories, the onset of a myriad of wrinkles is allowed even if this solution deviates from experimental evidence. If the value of $\alpha$ is increased, the results obtained with the different meshes considered are the same as in Fig. 4 and, therefore, they are not included here. 


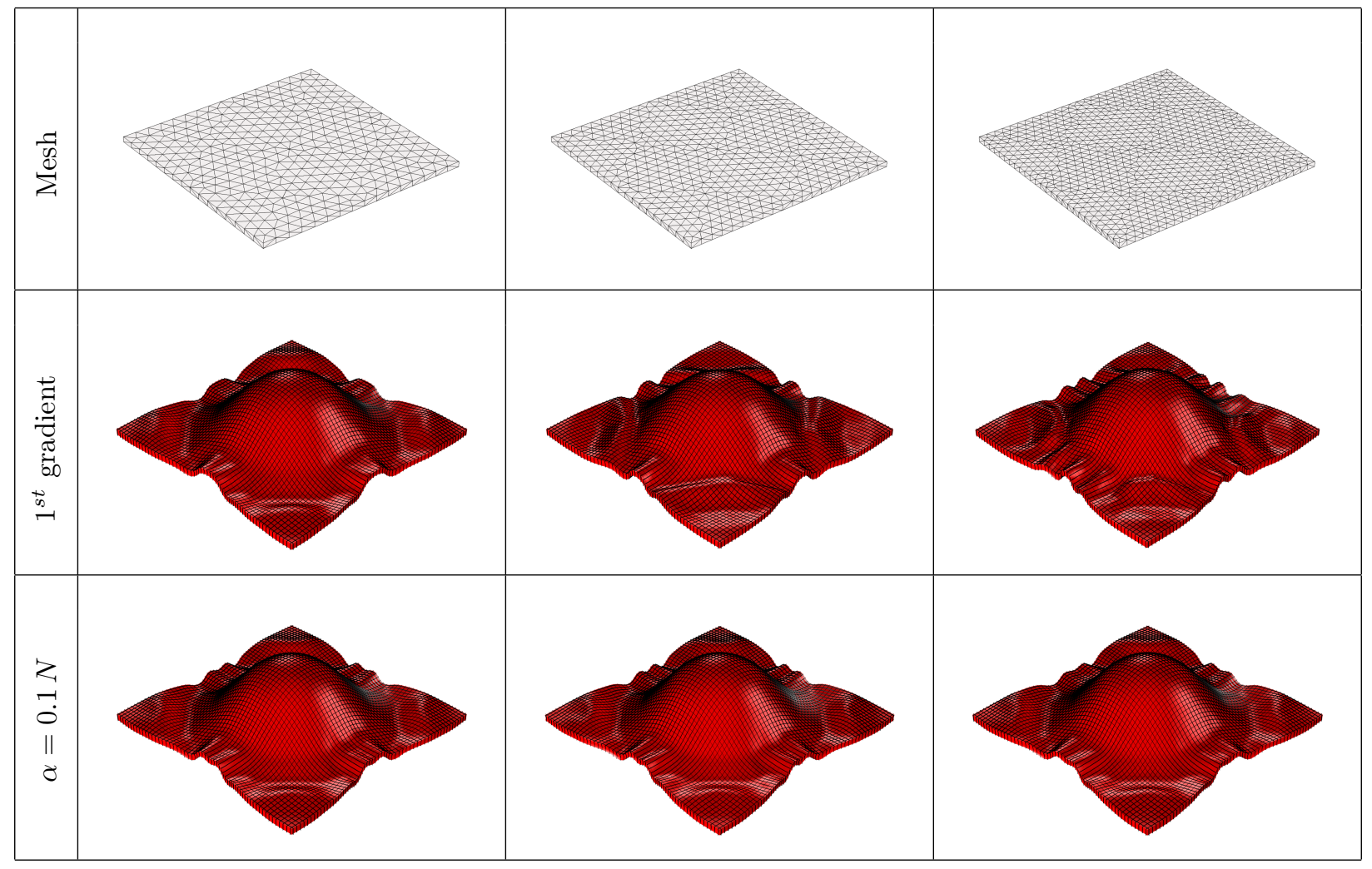

Figure 6: Mesh-dependency of the first and the second gradient model with shape functions with augmented continuity.

The presented results are very promising but, it must be noted that the augmented continuity shape functions are just a workaround for the real problem which is that of implementing robust finite elements for the simulation of woven composite reinforcements in view of structure design. The continuity of the derivatives is weakly imposed and it is, therefore, not strictly granted. A study on the validity of a model implementing the augmented continuity shape functions should be made, even if it seems that the presented model is reliable in the description of the analyzed phenomenon. Alternative methods to stabilize the solution can also be found in the literature [39] consisting in the insertion of small structural elements (such as beams) in the interior of the FE in the direction of the yarns, so indirectly accounting for their bending stiffness.

\subsubsection{Influence of cutting the corners on the onset of wrinkling for first and second gradient solutions}

During experimental testing, it is a spread routine to cut the corners of the specimen as shown in the first line of figure 7, see [15]. This change in the geometry can have an influence on the onset of wrinkling during the deep drawing of the fabric. It is possible to notice that cutting the corner leads to a slightly reduced amount of wrinkling in the first gradient model, while for the second gradient model the wrinkling is already not relevant and therefore almost no influence is seen by the cutting of the corners. The considerations concerning the dependence of the solution from the size of the mesh remain the same as in the previous subsections both for the first and second gradient case. 


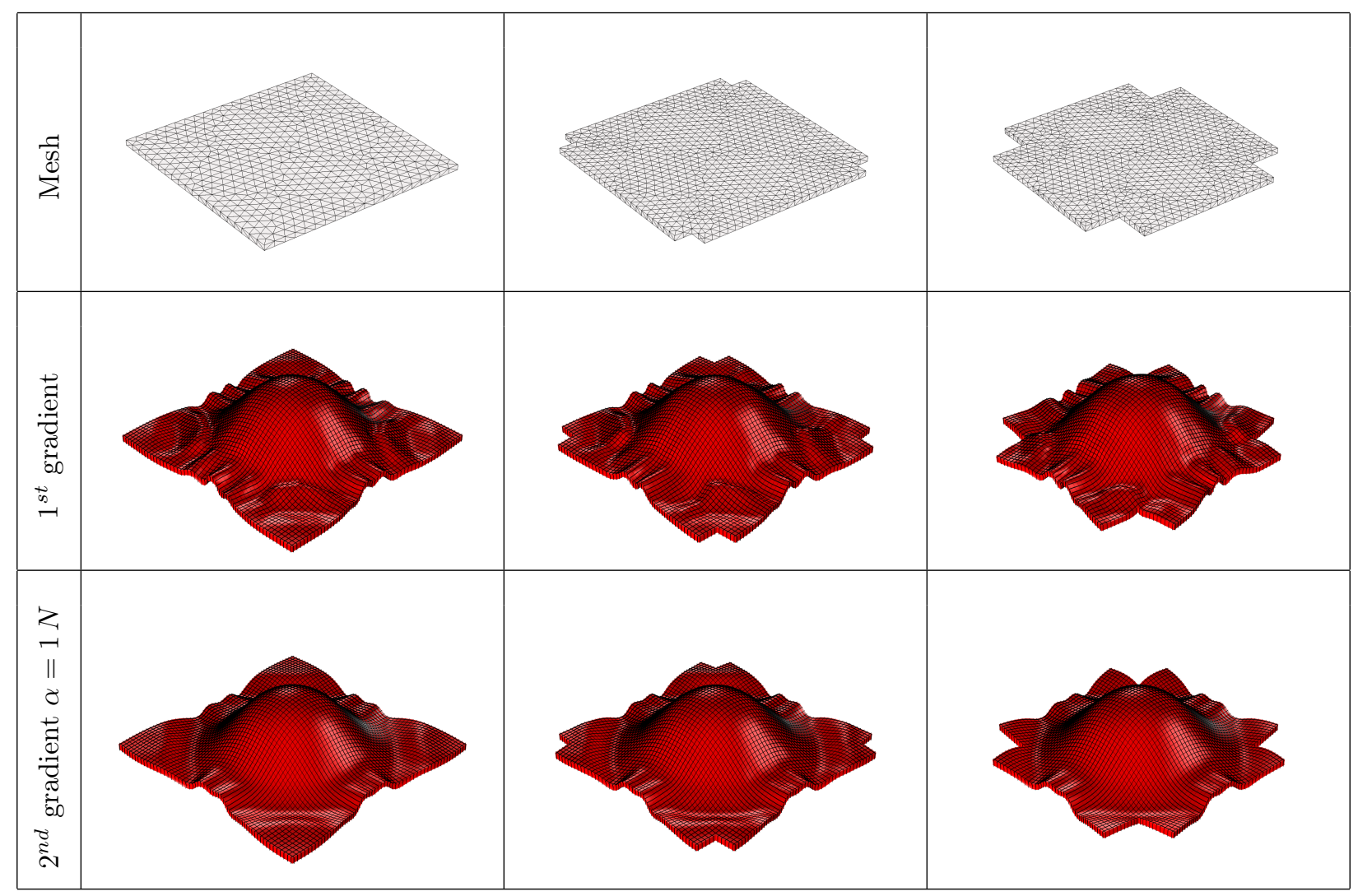

Figure 7: Influence of cutting the corners on the onset of wrinkling for the first and the second gradient model with shape functions with augmented continuity.

\section{Conclusions}

The woven fabrics posses a huge potential due to their very specific characteristics. However, there is still no common agreement concerning the approach to be adopted for the modeling of such materials during their dry preforming. The classical models used for the woven composite preforming fail to describe a wealth of observed experimental evidences. In particular, the description of the wrinkling phenomenon is one of the weakest points of such models.

The introduction of terms in the energy depending of higher order derivatives are useful to describe the deformation energy associated to the micro-structure. In the authors' opinion, this possibility is of fundamental importance for an accurate description of the preforming of the woven fabrics as well as for the control of the wrinkling phenomenon. In this paper, the results obtained with the introduction of an energy depending on the in-plane and out-of-plane curvature of the yarns are presented and it is shown how the onset of the wrinkling during the deep drawing can be controlled so as to reproduce real experimental evidence.

In the literature, second gradient models have already been used to describe specific experimental behaviors of fibrous composite reinforcements and this article provides additional evidences supporting the potential of the use of this type of models for such materials. It is the authors' belief that it should be considered a proven fact that the addition of simple second gradient energy terms could be critical to describe numerous observed phenomena such as the one analyzed here. However, the simple quadratic energy hereby used is not likely to be completely sufficient for the general description of the non-linear behavior of the fabrics, but it is descriptive enough to understand the usefulness of a second gradient model for such materials. Further studies are needed to find more complex non-linear second gradient constitutive laws fully descriptive of the mechanical behavior of the woven fabrics at very large strains and subjected to different boundary and/or loading conditions. 


\section{References}

[1] Yamina Aimène, Emmanuelle Vidal-Sallé, Benjamin Hagège, François Sidoroff, and Philippe Boisse. A hyperelastic approach for composite reinforcement large deformation analysis. Journal of Composite Materials, 44(1):5-26, 2010.

[2] Jean-Jacques Alibert, Pierre Seppecher, and Francesco dell'Isola. Truss modular beams with deformation energy depending on higher displacement gradients. Mathematics and Mechanics of Solids, 8(1):51-73, 2003.

[3] Holm Altenbach, Victor A. Eremeyev, Leonid P. Lebedev, and Leonardo A. Rendón. Acceleration waves and ellipticity in thermoelastic micropolar media. Archive of Applied Mechanics, 80(3):217-227, 2010.

[4] Ali Asghar Atai and David J. Steigmann. On the nonlinear mechanics of discrete networks. Archive of Applied mechanics, 67(5):303-319, 1997.

[5] Daniel Balzani, Patrizio Neff, Jörg Schröder, and Gerhard A. Holzapfel. A polyconvex framework for soft biological tissues. Adjustment to experimental data. International Journal of Solids and Structures, 43(20):6052-6070, 2006.

[6] Gabriele Barbagallo, Angela Madeo, Ismael Azehaf, Ivan Giorgio, Fabrice Morestin, and Philippe Boisse. Bias extension test on an unbalanced woven composite reinforcement: Experiments and modeling via a second-gradient continuum approach. Journal of Composite Materials, 2016.

[7] Jeffrey L. Bleustein. A note on the boundary conditions of Toupin's strain-gradient theory. International Journal of Solids and Structures, 3(6):1053-1057, 1967.

[8] Philippe Boisse, K. Buet, Alain Gasser, and Jean Launay. Meso/macro-mechanical behaviour of textile reinforcements for thin composites. Composites Science and Technology, 61(3):395-401, 2001.

[9] Philippe Boisse, A. Hakim Cherouat, Jean Claude Gelin, and Hamid Sabhi. Experimental study and finite element simulation of a glass fiber fabric shaping process. Polymer composites, 16(1):83-95, 1995.

[10] Philippe Boisse, Nahiène Hamila, F. Helenon, B. Hagege, and Jian Cao. Different approaches for woven composite reinforcement forming simulation. International Journal of Material Forming, 1(1):21-29, 2008.

[11] Philippe Boisse, Nahiène Hamila, Emmanuelle Vidal-Sallé, and François Dumont. Simulation of wrinkling during textile composite reinforcement forming. Influence of tensile, in-plane shear and bending stiffnesses. Composites Science and Technology, 71(5):683-692, 2011.

[12] Philippe Boisse, Bassem Zouari, and Jean-Luc Daniel. Importance of in-plane shear rigidity in finite element analyses of woven fabric composite preforming. Composites Part A: Applied Science and Manufacturing, 37(12):2201-2212, 2006.

[13] Philippe Boisse, Bassem Zouari, and Alain Gasser. A mesoscopic approach for the simulation of woven fibre composite forming. Composites Science and Technology, 65(3-4):429-436, 2005.

[14] Jian Cao, Remko Akkerman, Philippe Boisse, Julie Chen, H. S. Cheng, E. F. de Graaf, J. L. Gorczyca, Philip Harrison, Gilles Hivet, Jérôme Launay, Wonoh Lee, L. Liu, Stepan V. Lomov, Andrew C. Long, Emmanuel de Luycker, Fabrice Morestin, J. Padvoiskis, X. Q. Peng, James Sherwood, T. Stoilova, Xiaoming M. Tao, I. Verpoest, A. Willems, J. Wiggers, T. X. Yu, and B. Zhu. Characterization of mechanical behavior of woven fabrics: Experimental methods and benchmark results. Composites Part A: Applied Science and Manufacturing, 39(6):1037-1053, 2008.

[15] Adrien Charmetant, Jean Guillaume Orliac, Emmanuelle Vidal-Sallé, and Philippe Boisse. Hyperelastic model for large deformation analyses of 3D interlock composite preforms. Composites Science and Technology, 72(12):1352-1360, 2012.

[16] Adrien Charmetant, Emmanuelle Vidal-Sallé, and Philippe Boisse. Hyperelastic modelling for mesoscopic analyses of composite reinforcements. Composites Science and Technology, 71(14):1623-1631, 2011.

[17] Eugène Cosserat and François Cosserat. Théorie des corps déformables (engl. translation by D. Delphenich 2007, pdf available at http://www.uni-due.de/\%7ehm0014/Cosserat_files/Cosserat09_eng.pdf). 1909.

[18] Emmanuel de Luycker, Fabrice Morestin, Philippe Boisse, and David Marsal. Simulation of 3D interlock composite preforming. Composite Structures, 88:615-623, 2009.

[19] Emmanuel de Luycker, Jean Guillaume Orliac, Fabrice Morestin, Philippe Boisse, David Marsal, and Stephane Otin. Experimental and numerical analyses of 3D interlock composite preforming. International Journal of Material Forming, 3(1):719-722, 2010.

[20] Francesco dell'Isola, Giulio Sciarra, and Stefano Vidoli. Generalized Hooke's law for isotropic second gradient materials. Proceedings of the Royal Society A: Mathematical, Physical and Engineering Sciences, 465(2107):2177-2196, 2009.

[21] Francesco dell'Isola and David J. Steigmann. A two-dimensional gradient-elasticity theory for woven fabrics. Journal of Elasticity, 118(1):113-125, 2015.

[22] J. P. Dumont, Pierre Ladeveze, M. Poss, and Yves Remond. Damage mechanics for 3-D composites. Composite Structures, 8(2):119-141, 1987.

[23] Damien Durville. Numerical simulation of entangled materials mechanical properties. Journal of Materials Science, 40(22):5941-5948, 2005.

[24] Victor A. Eremeyev. Acceleration waves in micropolar elastic media. Doklady Physics, 50(4):204-206, 2005.

[25] Victor A. Eremeyev, Leonid P. Lebedev, and Holm Altenbach. Foundations of micropolar mechanics. Springer Berlin Heidelberg, 2013.

[26] Ahmed Cemal Eringen. Microcontinuum field theories. Springer-Verlag, New York, 1999.

[27] Manuel Ferretti, Angela Madeo, Francesco dell'Isola, and Philippe Boisse. Modeling the onset of shear boundary layers in fibrous composite reinforcements by second-gradient theory. Zeitschrift für Angewandte Mathematik und Physik, 65(3):587612,2014 
[28] Sébastien Gatouillat, Andrea Bareggi, Emmanuelle Vidal-Sallé, and Philippe Boisse. Meso modelling for composite preform shaping - Simulation of the loss of cohesion of the woven fibre network. Composites Part A: Applied Science and Manufacturing, 54:135-144, 2013.

[29] Paul Germain. The method of virtual power in continuum mechanics. Part 2: Microstructure. SIAM Journal on Applied Mathematics, 25(3):556-575, 1973.

[30] Albert Edward Green and Ronald S. Rivlin. Multipolar continuum mechanics. Archive for Rational Mechanics and Analysis, 17(2):113-147, 1964.

[31] Nahiène Hamila and Philippe Boisse. A meso-macro three node finite element for draping of textile composite preforms. Applied Composite Materials, 14(4):235-250, 2007.

[32] Eliza M. Haseganu and David J. Steigmann. Equilibrium analysis of finitely deformed elastic networks. Computational Mechanics, 17(6):359-373, 1996.

[33] Gerhard A. Holzapfel, Thomas C. Gasser, and Ray W. Ogden. A new constitutive framework for arterial wall mechanics and a comparative study of material models. Journal of Elasticity, 61(1-3):1-48, 2000.

[34] Mikhail Itskov and Nuri Aksel. A class of orthotropic and transversely isotropic hyperelastic constitutive models based on a polyconvex strain energy function. International Journal of Solids and Structures, 41(14):3833-3848, 2004.

[35] Wonoh Lee, J. Padvoiskis, Jian Cao, Emmanuel de Luycker, Philippe Boisse, Fabrice Morestin, J. Chen, and James Sherwood. Bias-extension of woven composite fabrics. International Journal of Material Forming, 1(SUPPL. 1):895-898, 2008.

[36] Angela Madeo, Gabriele Barbagallo, Marco Valerio d'Agostino, and Philippe Boisse. Continuum and discrete models for unbalanced woven fabrics. International Journal of Solids and Structures, 2016.

[37] Angela Madeo, Manuel Ferretti, Francesco dell'Isola, and Philippe Boisse. Thick fibrous composite reinforcements behave as special second-gradient materials: three-point bending of 3D interlocks. Zeitschrift für Angewandte Mathematik und Mechanik, 66(4):2041-2060, 2015.

[38] Sylvain Mathieu, Philippe Boisse, Nahiène Hamila, and Florent Bouillon. Locking and stability of 3D woven composite reinforcements. Key Engineering Materials, 611-612:292-299, 2014.

[39] Sylvain Mathieu, Nahiène Hamila, F. Dupé, C. Descamps, and Philippe Boisse. Stability of 3D Textile Composite Reinforcement Simulations: Solutions to Spurious Transverse Modes. Applied Composite Materials, 2016.

[40] Amin Mikdam, Ahmed Makradi, Said Ahzi, Hamid Garmestani, Dongsheng S. Li, and Yves Remond. Effective conductivity in isotropic heterogeneous media using a strong-contrast statistical continuum theory. Journal of the Mechanics and Physics of Solids, 57(1):76-86, 2009.

[41] Amin Mikdam, Ahmed Makradi, Said Ahzi, Hamid Garmestani, Dongsheng S. Li, and Yves Remond. Statistical continuum theory for the effective conductivity of fiber filled polymer composites: Effect of orientation distribution and aspect ratio. Composites Science and Technology, 70(3):510-517, 2010.

[42] Raymond David Mindlin. Micro-structure in linear elasticity. Archive for Rational Mechanics and Analysis, 16(1):51-78, 1964.

[43] Ben Nadler, Panayiotis Papadopoulos, and David J. Steigmann. Multiscale constitutive modeling and numerical simulation of fabric material. International Journal of Solids and Structures, 43(2):206-221, 2006.

[44] Ray W. Ogden. Non-linear elastic deformations, volume 1. 1984

[45] Victor G. Oshmyan, Stanislav A. Patlazhan, and Yves Remond. Principles of structural-mechanical modeling of polymers and composites. Polymer Science Series A, 48(9):1004-1013, 2006.

[46] Richard S. Parnas. Liquid Composite Molding. 2000.

[47] Juan Pazmino, Sylvain Mathieu, Valter Carvelli, Philippe Boisse, and Stepan V. Lomov. Numerical modelling of forming of a non-crimp 3D orthogonal weave E-glass composite reinforcement. Composites Part A: Applied Science and Manufacturing, 72:207-218, 2015.

[48] Wojciech Pietraszkiewicz and Victor A. Eremeyev. On vectorially parameterized natural strain measures of the non-linear Cosserat continuum. International Journal of Solids and Structures, 46(11-12):2477-2480, 2009.

[49] Gabbro Piola. Memoria intorno alle equazioni fondamentali del movimento di corpi qualsivogliono considerati secondo la naturale loro forma e costituzione. Modena, Tipi del R.D. Camera, 1846.

[50] Kevin D. Potter. The early history of the resin transfer moulding process for aerospace applications. 1999.

[51] Annie Raoult. Symmetry groups in nonlinear elasticity: an exercise in vintage mathematics. Communications on Pure and Applied Analysis, 8(1):435-456, 2008.

[52] Chris D. Rudd, Andrew C. Long, K. N. Kendall, and C. Mangin. Liquid moulding technologies: Resin transfer moulding, structural reaction injection moulding and related processing techniques. 1997.

[53] Jörg Schröder, Patrizio Neff, and Daniel Balzani. A variational approach for materially stable anisotropic hyperelasticity. International Journal of Solids and Structures, 42(15):4352-4371, 2005.

[54] Pierre Seppecher, Jean-Jacques Alibert, and Francesco dell'Isola. Linear elastic trusses leading to continua with exotic mechanical interactions. Journal of Physics: Conference Series, 319(1):012018, 2011.

[55] David J. Steigmann. Equilibrium of prestressed networks. IMA Journal of Applied Mathematics, 48(2):195-215, 1992.

[56] David J. Steigmann. Invariants of the stretch tensors and their application to finite elasticity theory. Mathematics and Mechanics of Solids, 7(4):393-404, 2002. 
[57] David J. Steigmann. Frame-invariant polyconvex strain-energy functions for some anisotropic solids. Mathematics and Mechanics of Solids, 8(5):497-506, 2003.

[58] Richard A. Toupin. Theories of elasticity with couple-stress. Archive for Rational Mechanics and Analysis, 17(2):85-112, 1964.

[59] Peng Wang, Xavier Legrand, Philippe Boisse, Nahiène Hamila, and Damien Soulat. Experimental and numerical analyses of manufacturing process of a composite square box part: Comparison between textile reinforcement forming and surface 3D weaving. Composites Part B: Engineering, 78:26-34, 2015.

[60] Bassem Zouari, Jean-Luc Daniel, and Philippe Boisse. A woven reinforcement forming simulation method. Influence of the shear stiffness. Computers \&S Structures, 84(5-6):351-363, 2006. 